\title{
Biofortification of field-grown cassava by engineering expression of an iron transporter and ferritin
}

\author{
Narayanan Narayanan1, Getu Beyene', Raj Deepika Chauhan', Eliana Gaitán-Solís', Jackson Gehan', \\ Paula Butts ${ }^{1}$, Dimuth Siritunga ${ }^{2}$, Ihuoma Okwuonu ${ }^{3}$, Arthur Woll ${ }^{4}{ }^{4}$, Dulce M. Jiménez-Aguilar ${ }^{5}$, \\ Erick Boy ${ }^{6}$, Michael A. Grusak [1] ${ }^{7}$, Paul Anderson' and Nigel J. Taylor (ib ${ }^{1 \star}$
}

\begin{abstract}
Less than $10 \%$ of the estimated average requirement (EAR) for iron and zinc is provided by consumption of storage roots of the staple crop cassava (Manihot esculenta Crantz) in West African human populations. We used genetic engineering to improve mineral micronutrient concentrations in cassava. Overexpression of the Arabidopsis thaliana vacuolar iron transporter VIT1 in cassava accumulated three- to seventimes-higher levels of iron in transgenic storage roots than nontransgenic controls in confined field trials in Puerto Rico. Plants engineered to coexpress a mutated $A$. thaliana iron transporter (IRT1) and $A$. thaliana ferritin (FER1) accumulated iron levels 7-18 times higher and zinc levels 3-10 times higher than those in nontransgenic controls in the field. Growth parameters and storage-root yields were unaffected by transgenic fortification in our field data. Measures of retention and bioaccessibility of iron and zinc in processed transgenic cassava indicated that IRT1+FER1 plants could provide $40-50 \%$ of the EAR for iron and $60-70 \%$ of the EAR for zinc in 1- to 6-year-old children and nonlactating, nonpregnant West African women.
\end{abstract}

Micronutrient deficiency poses a threat to human health worldwide. An estimated 161 million children under 5 years of age are stunted, partly because of hidden hunger, which occurs when foodstuffs lack essential vitamins and minerals ${ }^{1}$. In Nigeria, $75 \%$ of preschool children and $67 \%$ of pregnant women are anemic ${ }^{2}$, and $20 \%$ of children under 5 years of age have zinc deficiency ${ }^{3}$. Irondeficiency anemia affects the immune system, stunts growth and impairs cognitive development in children ${ }^{4}$. Zinc deficiency causes increased risk of death from diarrhea, stunting and hindered cognitive development ${ }^{4}$. Biofortification of staple food crops through biotechnology is one of several strategies for improving essential micronutrients in foods for at-risk populations ${ }^{5}$. Approximately 800 million people worldwide consume the tropical root crop cassava, and one-third of the sub-Saharan African population relies on cassava for more than $50 \%$ of their caloric intake ${ }^{6}$. Although cassava is an excellent source of carbohydrate, the storage roots provide inadequate levels of bioavailable iron and $\mathrm{zinc}^{5,7}$. A lack of genetic variation for mineral traits within the cassava germplasm ${ }^{8}$ makes breeding new lines with improved mineral content challenging. A genetic engineering strategy has been undertaken to increase iron and zinc concentrations in cassava storage roots?
Genetic engineering has been successfully applied to increase mineral concentrations in cereal crops, including rice. Iron concentrations in polished rice grains have been increased by overexpressing the soybean or rice storage protein ferritin ${ }^{10}$ and coexpressing Arabidopsis nicotianamine synthase, common bean ferritin and Aspergillus phytase $^{11}$. Overexpression of AtIRT1, AtNAS1 and bean FERRITIN in rice resulted in 3.8-fold higher iron and 1.8-fold higher zinc concentrations than in the wild-type control ${ }^{12}$. Recently, overexpression of the soybean ferritin SFER-H1 and rice nicotianamine synthase OsNAS2 has achieved dietary targets for both iron and zinc nutrition in rice grains ${ }^{13}$. Despite successes in rice, reports of engineering-improved mineral biofortification in dicotyledonous plants are rare and are mainly restricted to the model plant $A$. thaliana. Nongrass plants use a reduction-based mechanism for iron acquisition ${ }^{14}$ mediated by the plasma-membrane-bound oxidoreductase FRO2, and the ZIP-family transporter IRT1. Transgenic overexpression of the algal iron assimilatory protein FEA1 has resulted in a threefold increase in storageroot iron concentrations in greenhouse-grown cassava ${ }^{15}$, but these promising results were not maintained in field trials. Increased zinc concentrations in storage roots have been achieved by overexpression of the A. thaliana (At) zinc transporters AtZIP1 and AtMTP1, but shoot development in transgenic plants is impaired ${ }^{16}$.

We previously found that overexpression of the A. thaliana vacuolar iron transporter VIT1 in cassava results in a three- to four-times increase in iron concentration in storage roots compared with the concentrations in nontransgenic controls under greenhouse conditions $^{17}$. Here, we report that coexpression of a mutant $A$. thaliana iron transporter (IRT1) $)^{18}$ and ferritin (FER1) generates transgenic cassava plants that accumulate iron and zinc in storage roots to substantial levels in the human diet. Data from field-grown VIT1 and $I R T 1+$ FER1 transgenic lines in Puerto Rico field trials (2014-2017) indicate that both technologies result in cassava storage roots and foodstuffs with elevated iron and zinc levels that may beneficially affect the nutritional status of consumers.

We designed IRT1 and FER1 expression cassettes to improve mineral uptake, by placing AtIRT1 (ref. ${ }^{18}$ ) under control of the A14 promoter, and to store iron in plastids, by expressing AtFER1 (ref. ${ }^{19}$ ) under control of the patatin type 1 promoter (Supplementary Fig. 1a). Transgenic cassava plants of cultivar TME 204 coexpressing IRT1 and FER1 mRNA were established in the greenhouse. The shoot and

'Donald Danforth Plant Science Center, St. Louis, MO, USA. ${ }^{2}$ University of Puerto Rico Mayagüez, Puerto Rico, USA. ${ }^{3}$ National Root Crops Research Institute, Umudike, Nigeria. ${ }^{4}$ Cornell High Energy Synchrotron Source, Cornell University, Ithaca, NY, USA. ${ }^{5}$ USDA-ARS Children's Nutrition Research Center, Baylor College of Medicine, Houston, TX, USA. ${ }^{6}$ Harvest Plus/International Food Policy Research Institute, Washington, DC, USA. ${ }^{7}$ USDA-ARS Edward T. Schafer Agricultural Research Center, Fargo, ND, USA. *e-mail: ntaylor@danforthcenter.org 
storage-root growth phenotypes were similar for all transgenic and nontransgenic controllines during 16 weeks of growth (Supplementary Fig. 1b-g). The presence of IRT1 and FER1 transgenes in the leaves of 4-week-old plants was confirmed by PCR (Supplementary Fig. 2a). Southern blot analyses verified the integration of the IRT1 and FER1 transgenes at one or two copies of the transfer DNA (T-DNA; Supplementary Fig. 2b), and mRNA expression of IRT1 and FER1 was confirmed by RT-qPCR in leaves, fibrous roots and storage roots of transgenic plants (Supplementary Fig. 2c-h). Inductively coupled plasma optical emission spectroscopy (ICP-OES) analysis revealed that the storage roots of IRT1 + FER1 transgenic plants had five- to six-times-higher iron and zinc concentrations than the storage roots of nontransgenic controls (Supplementary Fig. 1j,k). The maximum iron accumulation reached $55 \pm 13 \mu \mathrm{g} / \mathrm{g}$ dry weight (DW; mean \pm s.d.) compared with $10 \pm 2 \mu \mathrm{g} / \mathrm{g}$ DW for storage roots of nontransgenic plants (Supplementary Fig. 1j), and $26 \pm 12 \mu \mathrm{g} / \mathrm{g}$ DW zinc compared with $5 \pm 1 \mu \mathrm{g} / \mathrm{g}$ DW zinc in the nontransgenic controls (Supplementary Fig. 1k). IRT1 + FER1 transgenic plants had leaf iron concentrations two to three times higher than those of nontransgenic controls (Supplementary Fig. 1h), but no increase in zinc concentration was observed in foliar tissues (Supplementary Fig. 1i). The total iron and zinc content was determined in leaves, petioles, stem, fibrous roots and storage-root peels to assess whether the elevated mineral levels in the storage-root parenchyma resulted from depletion in other organs. When assessed as whole plants, IRT1 + FER1 transgenic lines, compared with nontransgenic controls, showed significantly higher $(P \leq 0.01)$ total iron and zinc content, by up to five and two times, respectively (Supplementary Fig. 3a,b). The maximum total iron accumulation reached $7,059 \pm 204 \mu \mathrm{g}$ iron in transgenic line 8023-14 compared with $1,311 \pm 38 \mu \mathrm{g}$ iron in nontransgenic plants. The iron content increased in all organs except fibrous roots, and the greatest increase occurred in storage roots (Supplementary Fig. 3a). The zinc content was increased in storage roots, root peels and stems, but not in leaves, petioles or fibrous roots of IRT1 + FER1 plants (Supplementary Fig. 3b).

VIT1 and IRT1 + FER 1 transgenic lines were evaluated in confined field trials at Isabela field station, University of Puerto Rico (Supplementary Fig. 4). Over a 12-month trial period, no significant differences were found between VIT1 transgenic plants and nontransgenic controls for root or shoot biomass, storage-root dry-matter content, number of roots, harvest index or linamarin concentration (Fig. 1 and Supplementary Fig. 5). For IRT1 + FER1 transgenic plants, 12 of the 17 lines tested in the field generated storage-root yields (Fig. 1g) and shoot yields (Supplementary Fig. 6a) comparable to those of nontransgenic controls, and there were no significant differences in the number of storage roots, harvest index, dry matter or total linamarin concentration (Supplementary Fig. 6).

Mineral accumulation was determined in the storage roots of field-grown plants. We previously reported that cassava plants overexpressing AtVIT1 accumulated up to $48 \mu \mathrm{g} / \mathrm{g}$ DW iron in storage roots under greenhouse conditions, a level three to four times higher than that in nontransgenic controls ${ }^{17}$. Similar results were seen in field-grown materials, in which 10 of the 15 VIT1 lines had iron concentrations three to seven times higher, reaching a maximum of $60 \pm 7 \mu \mathrm{g} / \mathrm{g}$ DW (Fig. 2a). No elevation in zinc concentration was observed in the storage roots produced by VIT1 transgenic plants (Fig. 2b). All 17 IRT1 + FER1 transgenic lines grown in the field accumulated significantly elevated levels of iron $(P \leq 0.001)$ in their storage roots, which reached $130 \pm 39 \mu \mathrm{g} / \mathrm{g} D W$, an 18 -fold increase over the $7.2 \pm 3 \mu \mathrm{g} / \mathrm{g} \mathrm{DW}$ in nontransgenic controls (Fig. 2c). Fifteen $I R T 1+F E R 1$ transgenic lines also had significantly elevated levels of storage-root zinc $(P \leq 0.001)$, and line 8023-19 reached a maximum of $103 \pm 30 \mu \mathrm{g} / \mathrm{g} \mathrm{DW}$, a level ten times higher than that in nontransgenic controls (Fig. 2d). The elevated iron in these storage roots was positively correlated with an elevation of zinc concentration $(r=0.64$; Fig. $2 c, d)$. VIT1 transgenic plants showed a minor but significant elevation in copper, manganese and nickel concentrations (Supplementary Fig. 7), but the cadmium concentrations were below the detection limits. Likewise, IRT1 + FER1 plants had elevated copper and manganese concentrations (Supplementary Fig. 8), but the nickel and cadmium concentrations were below the detection limits (data not shown).

The IRT1 transporter would be expected to drive iron and zinc uptake from the soil, and FER1 would be expected to provide a sink for iron storage ${ }^{19}$. Native IRT1 is a high-affinity ferrous-iron transporter necessary for metal uptake and is upregulated under lowiron conditions ${ }^{20}$. The mutant version of IRT1 (IRT1 K146R K171R) that we used maintains an upregulated state under iron-abundant conditions, and efficacy has been demonstrated in A. thaliana ${ }^{18}$. We found that overexpression of mutant IRT1 drove elevated iron and zinc accumulation in a crop plant under iron-abundant field conditions $(83 \mu \mathrm{g} / \mathrm{g}$ DW iron; Supplementary Fig. 4a). IRT1 can transport manganese, cadmium and cobalt in addition to iron and zinc $^{21}$. Elevated concentrations of toxic heavy metals in biofortified foods is a safety concern. Field-grown cassava plants coexpressing $I R T 1+F E R 1$ accumulated elevated manganese and copper levels, but not to toxic levels (Supplementary Fig. 8a,b).

The cadmium concentrations in greenhouse growth medium and field soil were below detectable levels, a result that may explain why cadmium was not detected in the storage roots of transgenic cassava plants. The potential for IRT1 + FER1 plants to accumulate cadmium was further tested by growing the high-iron- and highzinc-accumulating line 8023-19 (Fig. 2c,d) in potting medium spiked with $10 \mu \mathrm{M}$ cadmium sulfate. Nontransgenic control plants accumulated undetectable cadmium in the control medium, with levels increasing to $0.8 \pm 0.3 \mu \mathrm{g} / \mathrm{g} \mathrm{DW}$ in leaves and $0.64 \pm 0.2 \mu \mathrm{g} / \mathrm{g}$ DW in storage roots, when grown in medium supplemented with cadmium sulfate. Transgenic plants also accumulated cadmium when grown in high-cadmium medium but did so at levels two to five times lower than those in the nontransgenic controls (Supplementary Fig. 9a,b). In the presence of high cadmium, transgenic plants accumulated less iron and zinc in their storage roots than when grown in medium without supplemental cadmium (Supplementary Fig. 9d,f), thus indicating possible competition among cadmium, iron and zinc transport in cassava. Cultivation of cassava plants on medium artificially supplemented with cadmium indicated that $I R T 1+F E R 1$ transgenic plants accumulated cadmium at levels lower than those in nontransgenic controls (Supplementary Fig. 9a,b), thus suggesting that IRT1 + FER1 transgenic plants would not pose a higher risk of cadmium toxicity than nonmodified plants if grown in high-cadmium soils.

We planted stake cuttings from nontransgenic controls, three VIT1 (8012-4, 8012-11 and 8012-18) and three IRT1 + FER1 (8023$8,8023-10$ and 8023-17) transgenic lines in the field to assess the stability of mineral enhancement across the vegetative cropping cycle (Supplementary Fig. 10). After 12 months of growth, the storage roots were harvested and analyzed. All VIT1 lines showed a significant $(P \leq 0.001)$ six- to seven-times-higher iron concentration than that in nontransgenic controls, reaching a maximum of $62 \pm 14 \mu \mathrm{g} / \mathrm{g}$ DW (Supplementary Fig. 11a), levels equivalent to those obtained in the first planting cycle (Fig. 2a). VIT1 transgenic plants also showed a minor but significant increase in zinc concentration (Supplementary Fig. 11b). Likewise, IRT1 + FER1 lines established from stake cuttings accumulated iron and zinc, reaching $80 \mu \mathrm{g} / \mathrm{g}$ DW and $60 \mu \mathrm{g} / \mathrm{g} \mathrm{DW}$, respectively, in their storage roots (Supplementary Fig. 11g,h), levels equivalent to the concentrations measured in the first cropping cycle (Fig. 2c,d). At the end of the second 12-month growing period, there were no significant differences between shoot and storage-root yields in two of the VIT1 transgenic lines (Supplementary Fig. 11c-f). Lower shoot and root yields were observed in IRT1+FER1 transgenic plants than in nontransgenic controls over the second cropping 

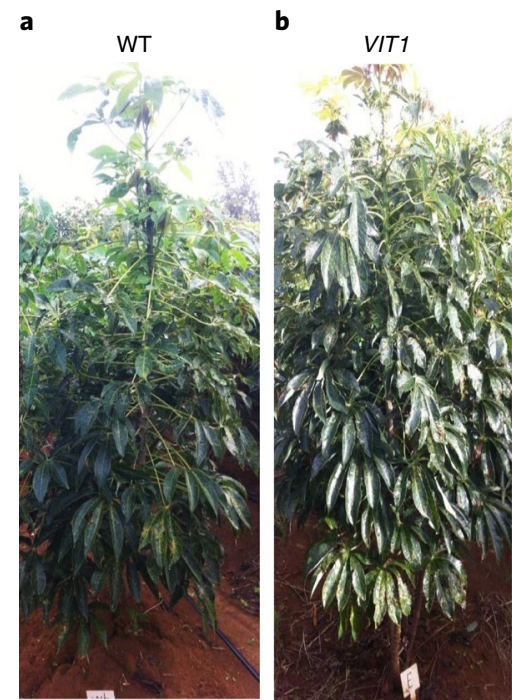

f

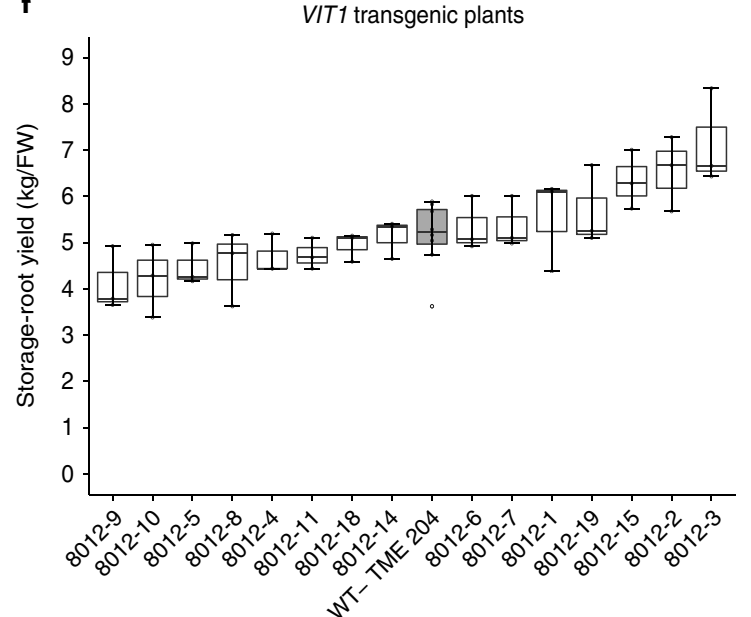

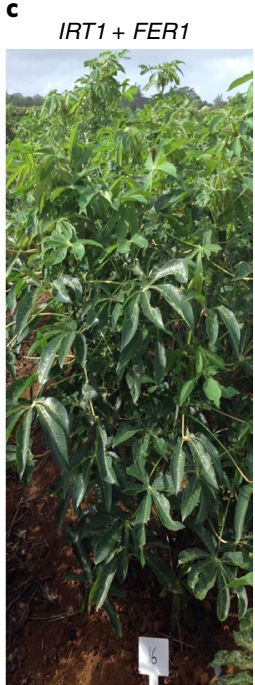

d WT-TME $204 \quad$ VIT1

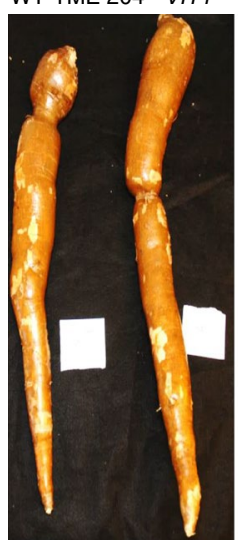

e WT-TME 204

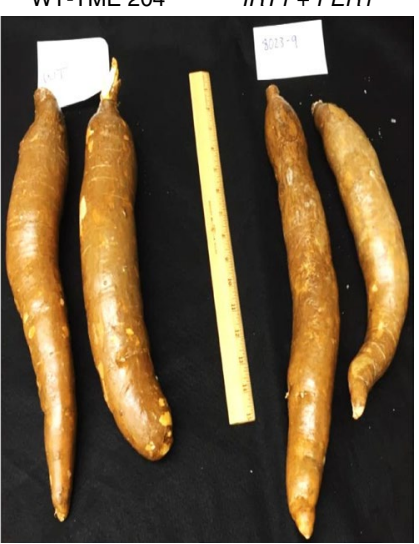

g

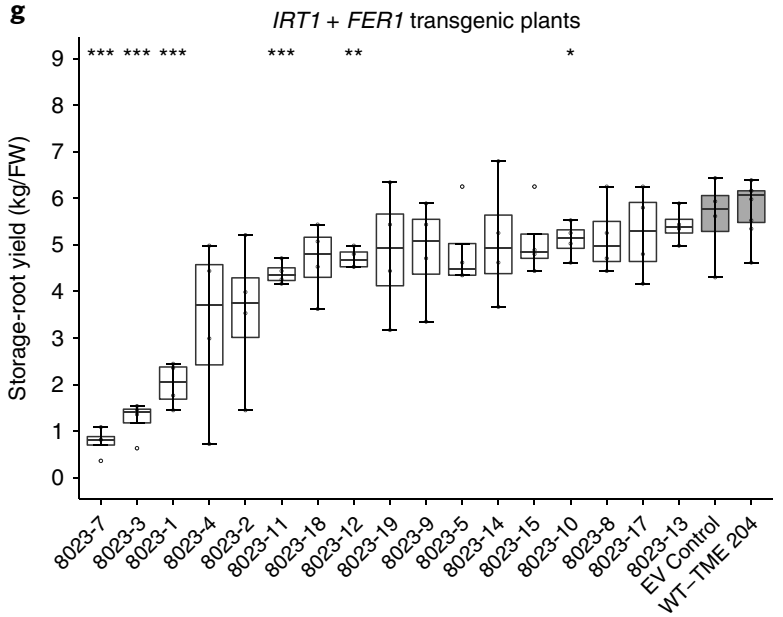

Fig. 1 | Agronomic yield of nontransgenic, VIT1 and IRT1+ FER1 transgenic cassava plants. a-e, Shoot phenotypes of 12-month-old nontransgenic control TME 204 (a), VIT1 (b) and IRT1 + FER1 (c) plants. Waxed storage roots of nontransgenic control and transgenic VIT1 (d) and IRT1 + FER1 (e) plants. f,g, Storage-root yields for VIT1 (f) and IRT1 + FER1 (g) transgenic plants, along with nontransgenic control. Box-and-whisker plots were constructed with the $\mathrm{R}$ package ggplot2. The upper whisker extends from the hinge to the largest value, no further than $1.5 \times$ the interquartile range (IQR, distance between the first and third quartiles) from the hinge. The lower whisker extends from the hinge to the smallest value, at most $1.5 \times$ the IQR of the hinge. Data beyond the ends of the whiskers are considered outlying points and are plotted individually. For VIT1, $n=9$ biologically independent plants ( 3 plants/replicate); for $I R T 1+F E R 1, n=4$ biologically independent plants. Statistical tests were performed with two-sided Student's $t$ test, relative to nontransgenic control. ${ }^{\star} P \leq 0.05 ;{ }^{\star \star} P \leq 0.01 ;{ }^{\star \star \star} P \leq 0.001$. WT, wild-type plants; EV control, empty-vector control plants.

cycle (Supplementary Fig. 11i-1). However, the storage-root yields observed for all three transgenic lines remained equivalent to historical averages achieved for the control cultivar TME 204, as measured across five confined field trials previously performed at the Isabela field station, Puerto Rico (Supplementary Fig. 12).

We analyzed the localization of iron and zinc in the stems and storage roots of transgenic plants by using elemental mapping through synchrotron X-ray fluorescence microscopy (XRF) ${ }^{22}$. On the basis of variations in tissue-section thickness and hydration, elemental and Compton-scattering XRF maps were obtained to compare and report elemental distributions (Supplementary Fig. 13a,b). The maps revealed that accumulated iron was associated with vascular tissues of the stem and storage roots in VIT1 and IRT1 + FER1 plants (Supplementary Fig. 14). VIT1 stems and storage roots showed strong localization of iron but minimal localization of zinc (Supplementary Fig. 14b,e), whereas strong colocalization of iron and zinc was seen in IRT1 + FER1 plants within the same tissue types (Supplementary Fig. 14c,f). In stems, this colocalization was associated with the stele and in the storage root with xylem vessels of the storage parenchyma (Supplementary Fig. 14c,f).

To be nutritionally useful, the increased mineral concentrations in transgenic plants must be retained in foodstuffs after processing. Therefore, we assessed the retention and bioaccessibility of iron and zinc in foods prepared from biofortified storage roots. Peeling and boiling of cassava is performed by many communities in East Africa ${ }^{23}$. VIT1 and IRT1 + FER1 transgenic storage-root parenchyma tissues showed no significant decrease in iron or zinc content after boiling (Supplementary Fig. 15a-c). Processing to produce the West African cassava foodstuffs gari and $f \mathrm{fu}^{23}$ is a more complex process involving chopping, soaking, fermenting, pressing and roasting. Iron retention in gari and fufu reached a minimum of $60 \%$ in both VIT1 and IRT1 + FER1 storage parenchyma compared with raw roots from the same plants (Fig. 3a,b), thus indicating the release of iron from the food matrix during processing. Zinc concentrations were $25-45 \%$ lower in gari and $55-60 \%$ lower in fufu than in unprocessed storage roots harvested from IRT1 +FER1 

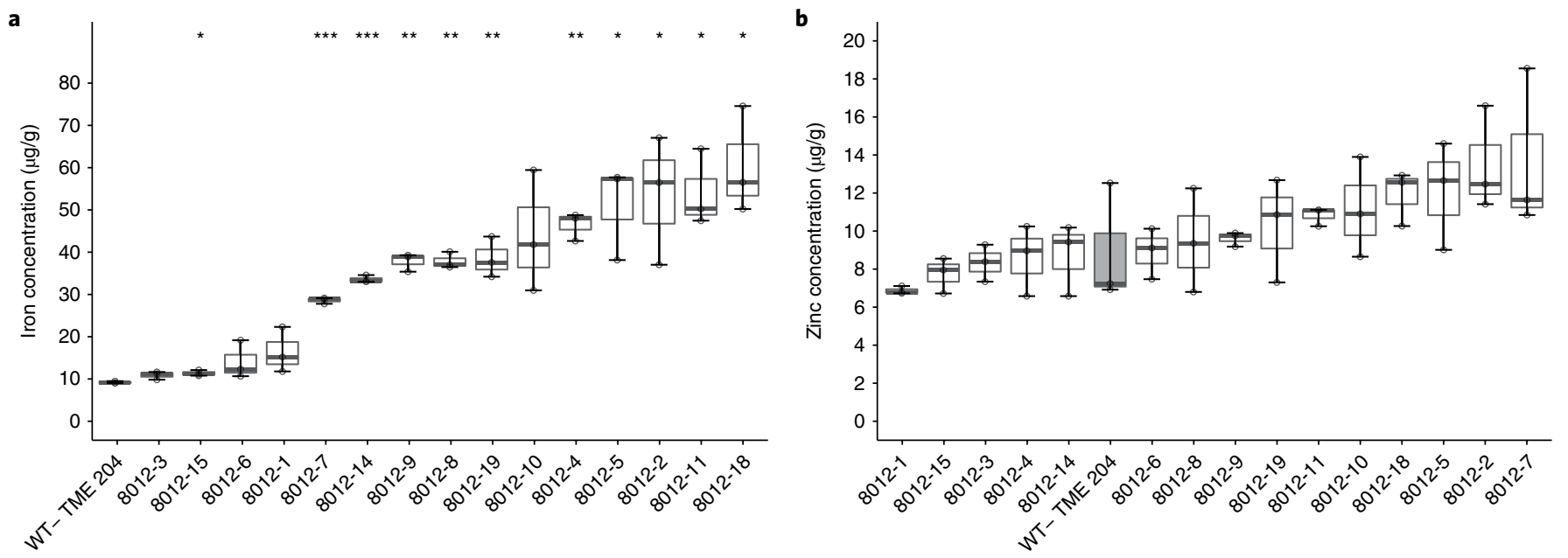

$I R T 1+$ FER1 transgenic lines
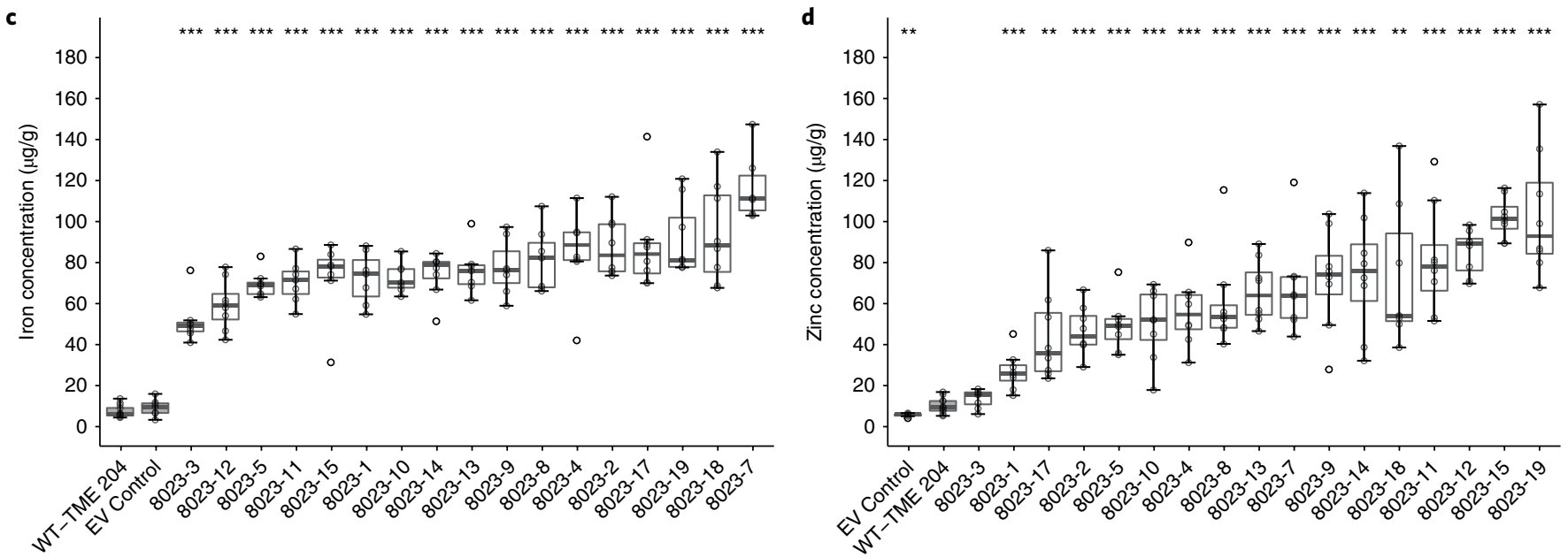

Fig. 2 | Storage-root mineral concentrations of VIT1 and IRT1 + FER1 transgenic cassava at harvest 12 months after planting under field conditions. $\mathbf{a}, \mathbf{b}$, Iron (a) and zinc (b) concentrations in storage roots of VIT1 transgenic cassava plants. $\mathbf{c}, \mathbf{d}$, Iron (c) and zinc (d) concentrations instorage roots of $I R T 1+F E R 1$ transgenic cassava plants. For VIT1, $n=9$ biologically independent plants (3 plants/replicate); for IRT1 + FER1, $n=4$ biologically independent plants. Box-and-whisker plots were constructed with the R package ggplot2. The upper whisker extends from the hinge to the largest value, no further than 1.5x the IQR from the hinge. The lower whisker extends from the hinge to the smallest value, at most $1.5 \times$ the IQR of the hinge. Data beyond the ends of the whiskers are considered outlying points and are plotted individually. Statistical tests were performed with two-sided Student's $t$ test, relative to nontransgenic control. ${ }^{\star} P \leq 0.05 ;{ }^{\star \star} P \leq 0.01 ;{ }^{\star \star \star} P \leq 0.001$.

transgenic lines (Fig. 3c). Importantly, however, equal rates of mineral loss were also found in gari and fufu prepared from storage roots of nontransgenic controls (Fig. $3 \mathrm{a}-\mathrm{c}$ ), thus indicating that the minerals in transgenic plants were retained at levels similar to the baseline levels present in nonmodified tissues. The steps in processing resulting in loss of iron and zinc are unknown, but a similar loss of iron has been reported during milling of rice, millet and wheat ${ }^{24}$, and in cooked cowpea meal ${ }^{25}$.

Bioaccessibility was assessed to determine the potential of availability of iron and zinc present within cassava foods for absorption in the gut after digestion. VIT1 transgenic storage roots had significantly higher iron bioaccessibility in uncooked roots (up to 1.5 times higher), processed gari (up to 2.8 times higher) and processed fufu (up to 3 times higher) than that of foods processed from the nontransgenic control (Fig. 3d). No differences were observed in zinc bioaccessibility in raw and processed fufu in the VIT1 transgenic storage roots and nontransgenic controls (Fig. 3e), whereas processed gari had significantly higher levels in three events and lower levels in two transgenic events, as compared with nontransgenic controls (Fig. 3e). No significant differences in iron or zinc bioaccessibility were detected in processed foods from IRT1 + FER 1 and nontransgenic control plants (Fig. 3f,g). Interestingly, VIT1 transgenic storage roots had significantly lower levels of both iron $(20-65 \%)$ and zinc $(15-65 \%)$ bioaccessibility in processed gari and fufu than in uncooked samples from the same plants (Fig. 3d,e). $I R T 1+F E R 1$ transgenic storage roots showed significantly higher levels (27-54\%) of iron bioacessibility in processed fufu (Fig. 3f) and significantly lower levels (18-36\%) of zinc bioaccessibility in processed gari than in uncooked samples (Fig. $3 \mathrm{~g}$ ). The significantly higher iron bioaccessibility in VIT1 transgenic plants than in nontransgenic controls (Fig. 3d) may have been due to an association of stored iron with soluble organic acids within the vacuole ${ }^{26}$, whereas the lack of differences in bioaccessibility in IRT1 +FER 1 transgenic plants relative to nontransgenic controls (Fig. 3f,g) may have resulted from iron stored as ferritin being less available for release from the storage-root tissues ${ }^{19}$.

The nutritional effects of consuming cassava storage roots biofortified by overexpression of VIT1 and IRT1 + FER 1 was assessed 

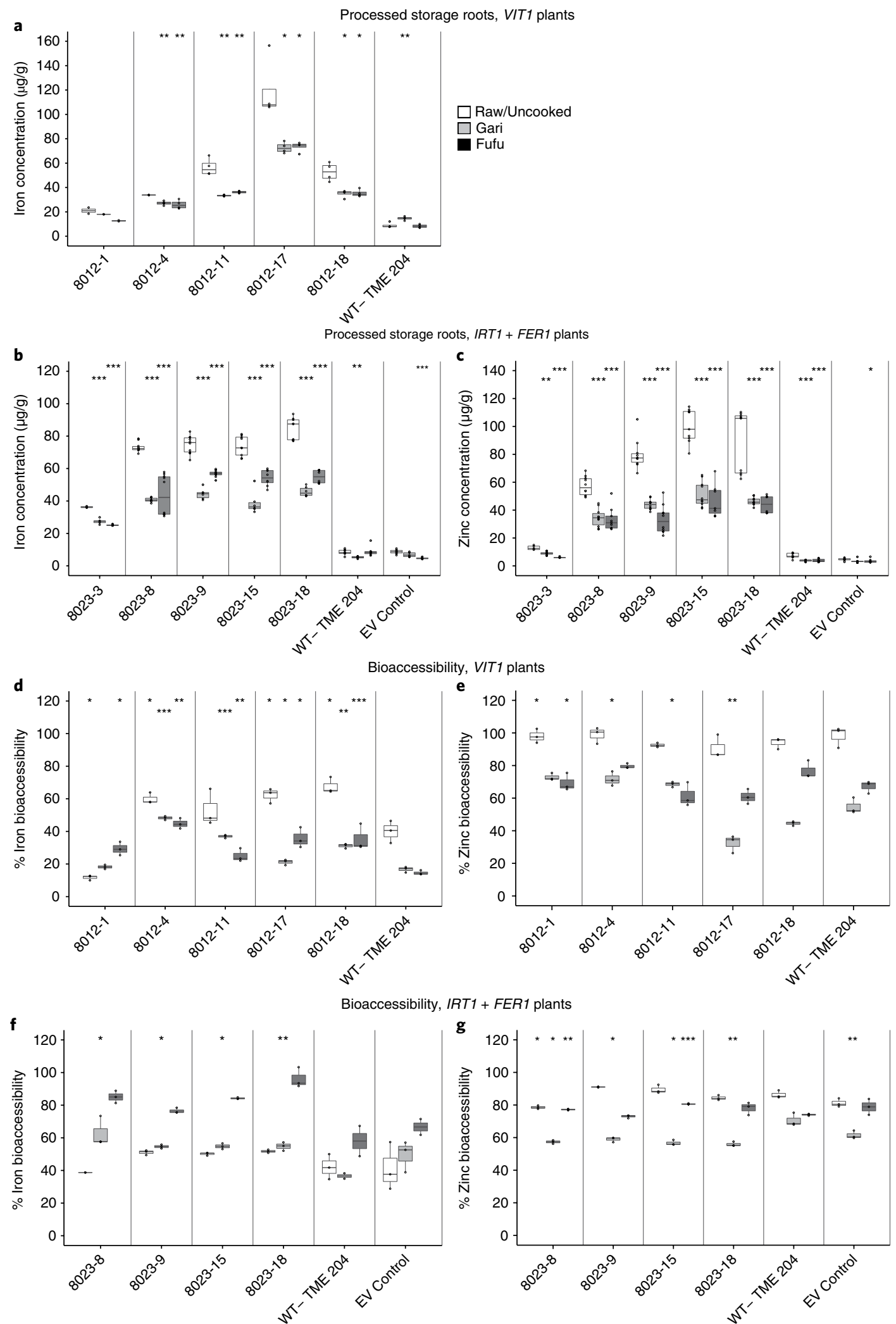

Fig. 3 | Mineral retention and bioaccessibility of processed VIT1 and IRT1+FER1 storage roots. a, Iron concentrations after processing of gari and fufu from the storage roots of VIT1 plants. b,c, Iron (b) and zinc (c) concentrations after processing of gari and fufu from storage roots of IRT1 + FER1 plants. $\mathbf{d}, \mathbf{e}$, Iron (d) and zinc (e) bioaccessibility of after processing of gari and fufu from storage roots of VIT1 plants. $\mathbf{f , g}$, Iron (f) and zinc (g) bioaccessibility after processing of gari and fufu from storage roots of IRT1 + FER1 plants. For VIT1, $n=3$ biologically independent plants; for IRT1 +FER1, $n=4$ biologically independent plants (2 technical replicates/plant). Box-and-whisker plots were constructed with the R package ggplot2. The upper whisker extends from the hinge to the largest value, no further than 1.5x the IQR from the hinge. The lower whisker extends from the hinge to the smallest value, at most $1.5 \times$ the IQR of the hinge. Data beyond the ends of the whiskers are considered outlying points and are plotted individually. Statistical tests were performed with two-sided Student's $t$ test, relative to raw storage roots within each line $(\mathbf{a}-\mathbf{c})$ or to nontransgenic control $(\mathbf{d}) .{ }^{\star} P \leq 0.05 ;{ }^{\star \star} P \leq 0.01$; ${ }^{\star \star \star} P \leq 0.001$. 

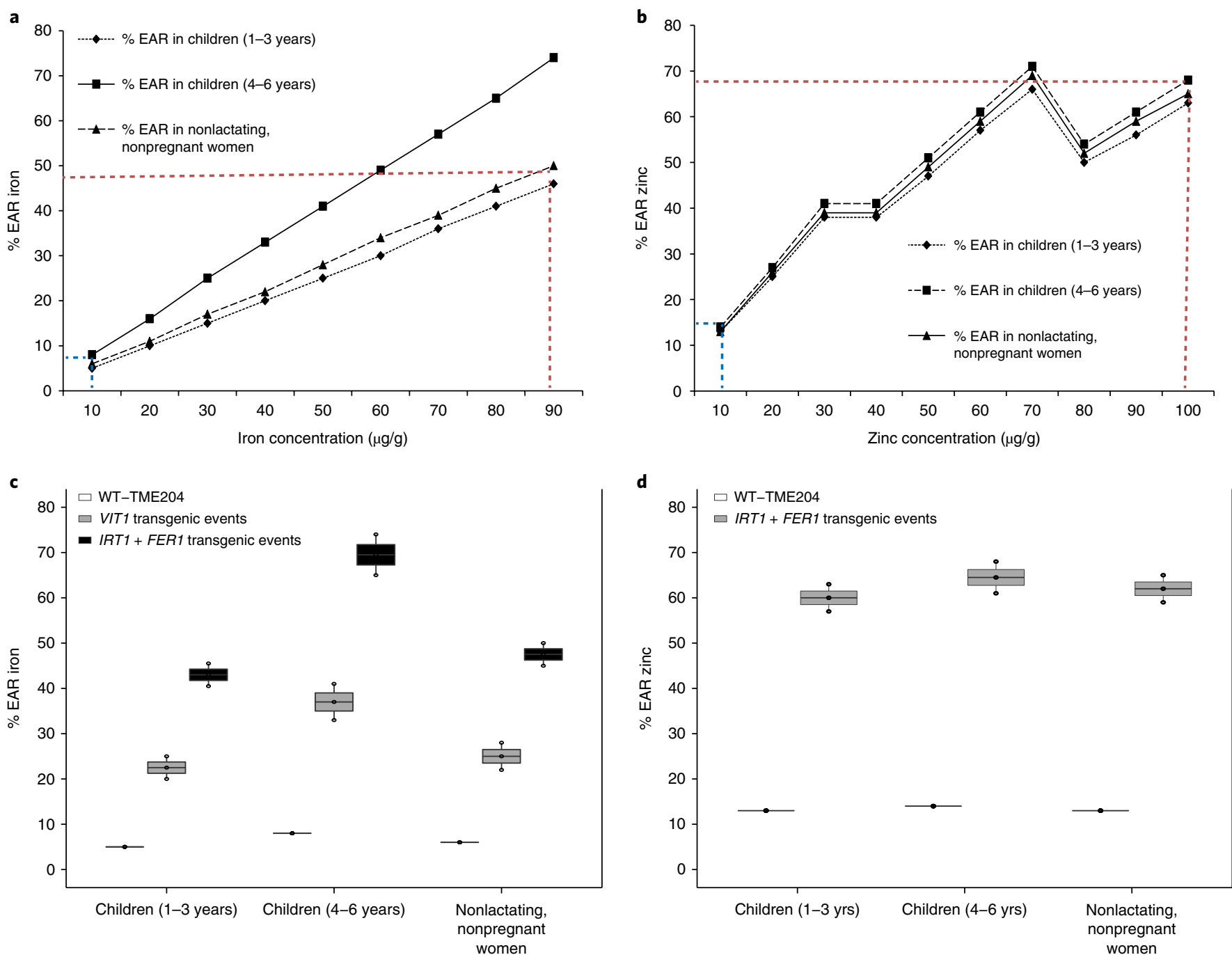

Fig. 4 | Contribution of biofortified transgenic cassava to EARs for iron and zinc. a,b, Graphical plots of iron (a) and zinc (b) concentrations from the transgenic cassava plants against the percentage EAR for children (1-3 years of age), children (4-6 years of age) and nonpregnant nonlactating women. Blue dashed line, percentage EAR calculated from processed food of baseline wild-type storage roots; red dashed line, percentage EAR calculated from processed food of transgenic cassava storage roots. c, Iron-biofortified VIT1 (containing an additional 40-50 $\mu \mathrm{g} / \mathrm{g}$ iron) or IRT1 + FERT (containing an additional $80-90 \mu \mathrm{g} / \mathrm{g}$ iron) cassava lines, showing the potential nutritional contribution toward the EARs for different demographic groups relative to

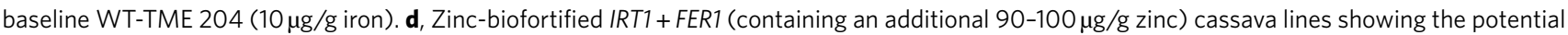
nutritional contribution toward the EARs for different demographic groups relative to baseline WT-TME 204 (10 $\mu \mathrm{g} / \mathrm{g}$ zinc).

by calculating their potential contribution to the $\mathrm{EAR}^{27}$ for iron and zinc. On the basis of consumption patterns in West Africa ${ }^{28}$, iron and zinc present in nonmodified cassava storage roots provide only $5-8 \%$ and $13-14 \%$ of the EAR for iron and zinc, respectively, for children 1-3 years old (Fig. 4a,d). Our data on mineral accumulation and retention enabled us to predict that IRT1 + FER1 transgenic plants of TME 204 contributed up to $40-50 \%$ of the EAR for iron for children (1-3 years old) and nonlactating, nonpregnant women, and $65-75 \%$ of the EAR for iron for children (4-6 years old) (Fig. $4 \mathrm{a}, \mathrm{c})$. In addition, IRT1 + FER 1 plants provided $60-70 \%$ of the EAR for zinc for children (1-3 years old), children (4-6 years old) and nonlactating, nonpregnant women (Fig. 4b,d).

Caco-2 studies were not undertaken on the iron- and zincbiofortified foods reported here, because it can assay for only iron bioavailability but does not generate data for zinc bioavailability or provide quantifiable values for mineral release from a digested food. Instead, an in vitro bioaccessibility assay was used to assess the release of iron and zinc from gari and fufu in transgenic versus nontransgenic derived foods, thus enabling calculation of the EAR for both minerals (Fig. 4).

In summary, of the 18 transgenic cassava plant lines coexpressing IRT1 + FER1 mRNA, 17 (94\%) attained nutritionally meaningful levels of iron and zinc that were able to provide $30-50 \%$ of the EAR for iron, and 15 attained levels were able to provide $40-70 \%$ of the EAR for zinc for children and nonlactating, nonpregnant women (Figs. 2 and 4). The success of our method surpasses that previously reported for approaches to engineer biofortification by using Agrobacterium-mediated integration into the plant genome. For example, in rice, more than 1,600 transgenic $\mathrm{T}_{0}$ lines were required to generate two low-T-DNA-copy lines with nutritionally meaningful iron and zinc elevation ${ }^{13}$. It is therefore possible that even lowlevel transgenic expression of mutated IRT1 may be effective for driving substantial uptake of iron and zinc from the growth medium into plant cells. The loss of iron and zinc during cassava processing occurred at levels higher than expected (Fig. 3), but, crucially, the loss rates were equivalent in transgenic and nontransgenic plants, 
and the available iron and zinc in processed foods remained meaningful in terms of nutritional value (Fig. 4). Factors such as the levels of vitamin $\mathrm{C}$ and organic acids present in the diet can increase mineral bioaccessibility and improve mineral absorption in the digestive tract $^{29}$. Cassava is not a recognized source of organic acids ${ }^{30}$, whereas vitamin C present in fresh storage roots is degraded up to $99 \%$ by commonly used processing techniques ${ }^{8}$. The nutritional value of the elevated iron and zinc present in biofortified cassava foodstuffs can be improved by the consumption of other foods in the diet that contain these absorption promoters. Therefore future studies should evaluate the effects of dietary vitamin $C$ levels on mineral micronutrient bioavailability in biofortified cassava foodstuffs.

The United Nations Sustainable Development Goals call for an end to global hunger and decreases in all forms of malnutrition by the year 2030 (ref. ${ }^{31}$ ). Nutritional security for the global population could be improved through biofortification of staple food crops such as cassava. We report that iron-enriched and iron- and zinc-enriched cassava storage roots can be grown in the field without decreases in yield. Our biofortified plant lines, or indeed other staple dicot crops such as sweet potato and potato, may be exploited as exemplars to improve the nutritional quality of cassava cultivars grown in different regions.

\section{Online content}

Any methods, additional references, Nature Research reporting summaries, source data, statements of data availability and associated accession codes are available at https://doi.org/10.1038/ s41587-018-0002-1.

Received: 9 January 2018; Accepted: 20 November 2018; Published online: 28 January 2019

\section{References}

1. de Onis, M. \& Branca, F. Childhood stunting: a global perspective. Matern. Child Nutr. 12 (Suppl. 1), 12-26 (2016).

2. World Health Organization. The Global Burden of Disease: 2004 Update (WHO Press, Geneva, 2008).

3. Federal Ministry of Health Nigeria Department of Community Development \& Population Activities Nutrition Division The Nigeria Food Consumption and Nutrition Survey (NFCNS). National guidelines on micronutrients deficiencies control in Nigeria (Abuja, Nigeria, 2005).

4. Black, R. E. et al. Maternal and child undernutrition: global and regional exposures and health consequences. Lancet 371, 243-260 (2008).

5. Hefferon, K. L. Nutritionally enhanced food crops; progress and perspectives. Int. J. Mol. Sci. 16, 3895-3914 (2015).

6. Howeler, R., Lutaladio, N. \& Thomas, G. Save and Grow: Cassava. A Guide to Sustainable Production Intensification (Food and Agriculture Organization of the United Nations, Rome, 2013).

7. Gegios, A. et al. Children consuming cassava as a staple food are at risk for inadequate zinc, iron, and vitamin A intake. Plant Foods Hum. Nutr. 65, 64-70 (2010).

8. Chavez, A. L. et al. Iron, carotene, and ascorbic acid in cassava roots and leaves. Food. Nutr. Bull. 21, 410-413 (2000).

9. de Valença, A. W., Bake, A., Brouwer, I. D. \& Giller, K. E. Agronomic biofortification of crops to fight hidden hunger in sub-Saharan Africa. Glob. Food Secur. 12, 8-14 (2017).

10. Oliva, N. et al. Large-scale production and evaluation of marker-free indica rice IR64 expressing phytoferritin genes. Mol. Breed. 33, 23-37 (2014).

11. Wirth, J. et al. Rice endosperm iron biofortification by targeted and synergistic action of nicotianamine synthase and ferritin. Plant. Biotechnol. J. 7, 631-644 (2009)

12. Boonyaves, K., Wu, T. Y., Gruissem, W. \& Bhullar, N. K. Enhanced grain iron levels in rice expressing an IRON-REGULATED METAL TRANSPORTER, NICOTIANAMINE SYNTHASE, and FERRITIN gene cassette. Front. Plant Sci. 8, 130 (2017)

13. Trijatmiko, K. R. et al. Biofortified indica rice attains iron and zinc nutrition dietary targets in the field. Sci. Rep. 6, 19792 (2016).

14. Rodríguez-Celma, J. \& Schmidt, W. Reduction-based iron uptake revisited: on the role of secreted iron-binding compounds. Plant Signal. Behav. 8, e26116 (2013).

15. Ihemere, U. E., Narayanan, N. N. \& Sayre, R. T. Iron biofortification and homeostasis in transgenic cassava roots expressing the algal iron assimilatory gene, FEA1. Front. Plant Sci. 3, 171 (2012).

16. Gaitán-Solís, E., Taylor, N. J., Siritunga, D., Stevens, W. \& Schachtman, D. P. Overexpression of the transporters AtZIP1 and AtMTP1 in cassava changes zinc accumulation and partitioning. Front. Plant Sci. 6, 492 (2015).
17. Narayanan, N. et al. Overexpression of Arabidopsis VIT1 increases accumulation of iron in cassava roots and stems. Plant Sci. 240, 170-181 (2015).

18. Kerkeb, L. et al. Iron-induced turnover of the Arabidopsis IRONREGULATED TRANSPORTER1 metal transporter requires lysine residues. Plant Physiol. 146, 1964-1973 (2008).

19. Briat, J. F., Duc, C., Ravet, K. \& Gaymard, F. Ferritins and iron storage in plants. Biochim. Biophys. Acta 1800, 806-814 (2010).

20. Vert, G. A., Briat, J. F. \& Curie, C. Dual regulation of the Arabidopsis high-affinity root iron uptake system by local and long-distance signals. Plant Physiol. 132, 796-804 (2003).

21. Korshunova, Y. O., Eide, D., Clark, W. G., Guerinot, M. L. \& Pakrasi, H. B. The IRT1 protein from Arabidopsis thaliana is a metal transporter with a broad substrate range. Plant Mol. Biol. 40, 37-44 (1999).

22. Punshon, T., Guerinot, M. L. \& Lanzirotti, A. Using synchrotron X-ray fluorescence microprobes in the study of metal homeostasis in plants. Ann. Bot. 103, 665-672 (2009)

23. Montagnac, J. A., Davis, C. R. \& Tanumihardjo, S. A. Nutritional value of cassava for use as a staple food and recent advances for improvement. Compr. Rev. Food Sci. Food Saf. 8, 181-194 (2009).

24. Oghbaei, M. \& Prakash, J. Effect of primary processing of cereals and legumes on its nutritional quality: a comprehensive review. Cogent Food Agric. 2, 1136015 (2016)

25. Pereira, E. J. et al. Effects of cooking methods on the iron and zinc contents in cowpea (Vigna unguiculata) to combat nutritional deficiencies in Brazil. Food Nutr. Res. 58, 20694 (2014).

26. Curie, C. et al. Metal movement within the plant: contribution of nicotianamine and yellow stripe 1-like transporters. Ann. Bot. 103, 1-11 (2009).

27. Kennedy, E. \& Meyers, L. Dietary reference intakes: development and uses for assessment of micronutrient status of women: a global perspective. Am. J. Clin. Nutr. 81, 1194S-1197S (2005).

28. Stephenson, K. et al. Consuming cassava as a staple food places children 2-5 years old at risk for inadequate protein intake, an observational study in Kenya and Nigeria. Nutr. J. 9, 9 (2010).

29. Etcheverry, P., Grusak, M. A. \& Fleige, L. E. Application of in vitro bioaccessibility and bioavailability methods for calcium, carotenoids, folate, iron, magnesium, polyphenols, zinc, and vitamins $\mathrm{B}(6), \mathrm{B}(12), \mathrm{D}$, and $\mathrm{E}$. Front. Physiol. 3, 317 (2012).

30. Organisation for Economic Co-operation and Development. Consensus document on compositional considerations for new varieties of CASSAVA (Manihot esculenta Crantz): key food and feed nutrients, anti-nutrients, toxicants and allergens (Series on the Safety of Novel Foods and Feeds No. 18, ENV/JM/MONO(2009)44) (Organisation for Economic Co-Operation and Development, Paris, 2009).

31. United Nations. Sustainable Development Goal 2: end hunger, achieve food security and improved nutrition and promote sustainable agriculture. United Nations Sustainable Development Knowledge Platform https://sustainabledevelopment.un.org/sdg2 (2017).

\section{Acknowledgements}

The authors thank M. Wu, A. Boyer, C. Albin, V. Carballo-Portela, A. Rai, J. Ma, T. Jones D. Posey and J. Winch at DDPSC for technical assistance. C. Luebbert from DDPSC is thanked for data analysis and box-plot production in R-Studio. L. Smieska (Cornell High Energy Synchrotron Source) is thanked for assistance in synchrotron X-ray fluorescence spectroscopy. We acknowledge E. Connolly (University of South Carolina) for the mutated version of AtIRT1 and R. Sayre (University of New Mexico) for the A14 promoter. We thank P. Raymond for statistical analysis, M. L. Guerinot for performing an advisory role and I. Baxter for critical reading of the manuscript.

\section{Author contributions}

E.G.-S., N.N. and P.A. conceived strategies for mineral uptake. N.N., G.B. and N.J.T. conceived and designed experiments. G.B. designed and constructed vectors for transformation. R.D.C. and N.J.T. generated the transgenic plants. J.G., N.N. and G.B. performed molecular analysis. N.N., P.B., J.G. and M.A.G. performed trait analysis. N.N. analyzed datasets. D.S., N.N., R.D.C., P.B. and J.G. designed and performed the field trials. A.W. and N.N. designed and performed X-ray synchrotron experiments. I.O., J.G., N.N., D.M.J.-A., M.A.G. and N.N. processed foodstuffs and performed retention and bioaccessibility studies. E.B. and N.N. calculated EARs. N.N., N.J.T., G.B. and M.A.G. wrote the manuscript.

\section{Competing interests}

This work was supported by the Bill \& Melinda Gates Foundation (BMGF) through the Global Challenges for Global Health Program (grant OPPGD1484) and the US Department of Agriculture, Agricultural Research Service, cooperative agreement 58-6250-0-008 (to M.A.G.). CHESS is supported by NSF award DMR-1332208. The contents of this publication do not necessarily reflect the views or policies of the US Department of Agriculture, nor does mention of trade names, commercial products or organizations imply endorsement by the US Government. BMGF had no role in the study design, collection, analysis or interpretation of data, in the writing of the report or in the decision to submit the article for publication. 


\section{Additional information}

Supplementary information is available for this paper at https://doi.org/10.1038/ s41587-018-0002-1.

Reprints and permissions information is available at www.nature.com/reprints. Correspondence and requests for materials should be addressed to N.J.T.

Publisher's note: Springer Nature remains neutral with regard to jurisdictional claims in published maps and institutional affiliations.

(C) This is a U.S. government work and not under copyright protection in the U.S.; foreign copyright protection may apply 2019
Open Access This article is licensed under a Creative Commons Attribution 4.0 International License, which permits use, sharing, adaptation, distribution and reproduction in any medium or format, as long as you give appropriate credit to the original author(s) and the source, provide a link to the Creative Commons license, and indicate if changes were made. The images or other third party material in this article are included in the article's Creative Commons license, unles indicated otherwise in a credit line to the material. If material is not included in the article's Creative Commons license and your intended use is not permitted by statutory regulation or exceeds the permitted use, you will need to obtain permission directly from the copyright holder. To view a copy of this license, visit http://creativecommons.org/licenses/by/4.0/. 


\section{Methods}

Generation of transgene constructs, plant transformation and plant materials. The binary vector p 8023 bearing dual expression cassettes for A. thaliana ironregulated transporter (AtIRT1, At4g19690.2) and ferritin 1 (AtFER1, At5g01600) was generated in the 55000 binary vector ${ }^{32}$. The amino acid sequence information for mutated AtIRT1 (ref. ${ }^{18}$ ) in binary vector pELC (provided by E. Connolly, University of South Carolina) was used to commercially synthesize this sequence after M. esculenta codon optimization. Both the promoter and the 3' UTR for driving expression of AtIRT1 were obtained from Arabidopsis cysteine/histidinerich C1 domain-containing protein (At5g43040.1) also known as A14 (ref. ${ }^{33}$ ). The $A$. thaliana ferritin 1 (AtFER1, At5G01600) expression cassette was generated with the type I patatin promoter and patatin $3^{\prime}$ UTR. Both AtFER1 and AtIRT1 expression cassettes were cloned into the binary vector p5000 (ref. $\left.{ }^{32}\right)$. The resulting binary vector bearing the T-DNA with AtIRT1, AtFER1 and the plant selectable marker $n p t I I$ driven by the duplicated CaMV 35 S promoter was named $\mathrm{p} 8023$. This construct was electroporated into Agrobacterium strain LBA4404 and used for transformation of cassava cultivar TME 204, as described earlier ${ }^{34}$. The mutated version of AtIRT1 is referred to as IRT1 herein for simplicity.

Molecular characterization of in vitro- and greenhouse-grown transgenic plants. Total RNA was extracted from $50 \mathrm{mg}$ of leaves obtained from 4 - to 6-week-old in vitro-grown cassava plantlets with a Promega RNA isolation kit, as described earlier ${ }^{17}$ and RT-PCR reactions were performed with AtIRT1- and AtFER1-specific primers (Table 1). For RT-qPCR, total RNA was extracted from $50 \mathrm{mg}$ of leaves, fibrous roots and lyophilized storage roots obtained from 16-weekold greenhouse-grown plants ${ }^{17}$, and RT-qPCR reactions were performed with AtIRT1-and AtFER1-specific primers (Table 1). Transcript levels in transgenic and wild-type plants were normalized to the expression level of protein phosphatase $2 \mathrm{~A}$ with the relative standard-curve method ${ }^{17}$

Plant establishment and growth in the greenhouse. In vitro transgenic and wild-type plantlets were planted in Fafard 51 mixture potting compost (Conrad Fafard) and established in the greenhouse $e^{35}$. For all experiments, eight independent transgenic plant lines were established, and plants were grown at $32^{\circ} \mathrm{C} / 27^{\circ} \mathrm{C}$ (day/ night) with $70-95 \%$ relative humidity. Plants were watered with reverse-osmosis water two or three times per day, as required, and fertilized twice weekly with Jack's professional fertilizer (JR Peters) at a rate of $100 \mu \mathrm{g} / \mathrm{g} \mathrm{DW}$ (ref. ${ }^{35}$ ).

Determination of total biomass in the greenhouse. Total biomass was determined for transgenic and wild-type plants after 16 weeks of growth in the greenhouse. Senescent leaves were collected in brown bags on alternate days over the 16 -week period and dried for $4-5 \mathrm{~d}$ in an oven at $60^{\circ} \mathrm{C}$. After 16 weeks, fresh weights of leaves, petioles, stems, fibrous roots, storage-root peels and storageroot parenchyma were recorded. Collected tissues were dried as described above. Samples of storage-root parenchyma (20-25 g fresh weight) were placed in $50-\mathrm{mL}$ conical tubes, frozen at $-80^{\circ} \mathrm{C}$ for $2-3 \mathrm{~h}$ and freeze-dried with a lyophilizer (Freezone18, Labconco) for at least $48 \mathrm{~h}$. Freeze-dried storage-root samples were weighed to determine dry-matter content, and total biomass per plant was calculated.

Determination of T-DNA copy number. T-DNA copy number was determined by extraction of genomic DNA from 1-2 $g$ of young leaves obtained from 4 - to 6-week-old greenhouse-grown transgenic and control plants with the CTAB protocol. Genomic DNA $(20 \mu \mathrm{g})$ from each sample was digested with 80 units of SpeI (New England BioLabs) overnight, subjected to electrophoresis on a $1 \%$ agarose gel and transferred with standard procedures. A $2 \times 35$ S promoter $(50 \mathrm{ng})$ was used as a probe. Hybridization and washing procedures were performed as previously described ${ }^{34}$.

Growth of transgenic plants in medium spiked with cadmium. Plants were treated with cadmium by transferring wild-type plants and plants transgenic for p8023 into 3-inch pots, weaning in a mist chamber for 1 week (ref. ${ }^{35}$ ) and growing on an open bench at $26^{\circ} \mathrm{C} / 25^{\circ} \mathrm{C}$ (day/night) for 2 weeks. After 4 weeks, plants were transferred to a growth chamber and maintained under a $16 \mathrm{~h} / 8 \mathrm{~h}$ photoperiod at a $35^{\circ} \mathrm{C} / 30^{\circ} \mathrm{C}$ (day/night) temperature cycle and $80-90 \%$ relative humidity for 13 weeks. Plants were watered with reverse-osmosis water two or three times per day, as required, and fertilized twice weekly with Jack's professional fertilizer (JR Peters) at a rate of 100 p.p.m. (ref. ${ }^{35}$ ). One set of plants was treated with $10 \mu \mathrm{M} \mathrm{CdSO}_{4}$ added along with the fertilizer twice per week, and the other was maintained as a control with a $0 \mu \mathrm{M}$ cadmium treatment. Four biological replicates were maintained for each control and transgenic plant line. After 13 weeks, leaves and storage roots were harvested and processed for measurement of minerals by ICP-OES.

Establishment and execution of confined field trials. Confined field trials were conducted at the Isabela Agriculture Research Station, Puerto Rico, Mayaguez. Soil samples ( $250 \mathrm{~g})$ were obtained from $10 \mathrm{~cm}$ below the soil surface with a wooden spatula, and chemical analysis was performed by A\&L Analytical Laboratories (Supplementary Fig. 4). In vitro plantlets were established in 50-mL conical tubes
Table 1 | Primers used in the study and their purposes

\begin{tabular}{lll} 
Name & Sequence & Purpose \\
\hline AtIRT1 & F, 5'-GCTTCGGACTTGTAAGATTCATCAGA-3' & RT-PCR \\
& R, 5'-TCATTCTGTTGTGATCGGACTTTCC-3' & \\
AtFER1 & F, 5'-TGAGACGATAGGGTGGAGTTTCAC-3' & RT-PCR \\
& R, 5'-ACCGGAGTCGTGTTCCAGCCTT-3' & \\
Cassava & F, 5'-GATCCTACTGGGAAGTACATTGG-3' & RT-PCR \\
tubulin & R, 5'-CTGCATTCTCCACCAACTGA-3' & \\
At/RT1 & F, 5'-TCCATCAGCTTCGGACTTGTAAGA-3' & RT-qPCR \\
& R, 5'-GGAGGAATGTCCATTATCGCCA-3' & \\
AtFER1 & F, 5'-GAGGTTGAAATACTTAAATGCGTGC-3' & RT-qPCR \\
& R, 5'-TAAGGATGATCGGCAAAGGCCA-3' & \\
$\begin{array}{l}\text { Cassava } \\
\text { protein } \\
\text { phosphatase } \\
\text { 2A }\end{array}$ & F, 5' 5 $^{\prime}$-TGCAAGGCTCACACTTTCATC-3' & RT-qPCR \\
\hline
\end{tabular}

$F$, forward; $R$, reverse

and shipped from DDPSC, St. Louis, to the University of Puerto Rico. Plantlets were transferred to Rain Forest potting soil (Sungro) in 4-inch pots and hardened for 1 month under light shelves (12 h light), followed by an additional 1 month in a glasshouse before being planted in the field. Confined field trials were established with a randomized block design with three replicates and six plants per line per replicate (Supplementary Fig. 4). A distance of $1.5 \mathrm{~m}$ was maintained between plants across all plants in the trial, which in turn were surrounded by a single row of nontransgenic wild-type plants. Drip irrigation was used in the dry season; otherwise, the trials were rain fed. The final harvest was performed 12 months after planting, and storage roots were harvested manually. At harvest, agronomic traits including shoot biomass, number of roots and root yield were determined. The harvest index was calculated as the storage root fresh weight divided by the total fresh weight of storage roots plus shoot biomass on a per-plant basis. Drymatter content was assessed by lyophilization of $35 \mathrm{~g}$ fresh weight of storage-root parenchyma for $2 \mathrm{~d}$. Three storage roots per transgenic line for each field-plot replicate were cleaned, dried, waxed and shipped to DDPSC, St. Louis. After receipt, the storage roots were peeled, chopped with ceramic knives and lyophilized as previously described ${ }^{17}$, in preparation for elemental and bioaccessibility analysis. Stake-derived field trials were established by obtaining stem cuttings from nontransgenic controls, three VIT1 (8012-4, 8012-11 and 8012-18) and three IRT1 + FER1 (8023-8, 8023-10 and 8023-17) transgenic lines at the time of harvest, 12 months after planting from in vitro plants. Stake cuttings were obtained from wood-stem material 6.0-8.0 inches in length and 1.5-2.0 inches in diameter, comprising five to seven nodes. Stakes were used to establish a randomized block design with four replicates and 20 plants per line per replicate. Other conditions remained the same as described above.

Measurement of mineral concentrations. All plant tissues were harvested and dried for $48 \mathrm{~h}$ in a $60^{\circ} \mathrm{C}$ oven. Two subsamples ( $\sim .5 \mathrm{~g}$ dry weight each) of ground, homogenized dried tissue were predigested overnight with $3 \mathrm{~mL}$ of ultrapure nitric acid in borosilicate glass tubes. Samples were digested as previously described ${ }^{36}$, and elemental concentrations were determined by ICP-OES (CIROS ICP Model FCE12; Spectro). Peach-leaf standards (SRM 1547 A; National Institute of Standards and Technology) were digested and analyzed along with each run of experimental samples to verify reliability of the procedures and analytical measurements; all values for peach-leaf standards were within their certified range. Mineral-content determinations were calculated by multiplication of each sample concentration by the total dry weight of that tissue sample.

Mineral analysis by synchrotron X-ray fluorescence spectroscopy ( $\mu$-XRF). Synchrotron-based XRF was used to obtain elemental concentrations and Compton scattering at the F3 station of the Cornell High Energy Synchrotron Source (CHESS), Cornell University. A double-crystal Si (220) monochromator was used to select an incident beam energy of $11.94 \mathrm{keV}\left(\Delta E / E \sim 10^{-4}\right)$, and a single-bounce monocapillary lens (capillary PeB605) ${ }^{37}$ was used to focus the beam to a $20-\mu \mathrm{m}$-diameter spot. For scans, stem and storage-root thin cross-sections $(0.2-0.3 \mathrm{~mm}$ thick) were obtained from live-tissue samples, weighed and mounted between one layer of $6.5-\mu \mathrm{m}$ Kapton polyimide film and a layer of $25-\mu \mathrm{m}$ Kapton tape, such that the thinner layer faced the detector. Fluorescence was measured with a 384-element Maia detector placed upstream of the sample and operating in backscatter geometry ${ }^{38}$. XRF images were obtained by continuous scanning of 
the sample horizontally across the beam, with typical integration times of $0.005 \mathrm{~s}$ / pixel. For petioles, stems and storage roots, two sections were obtained from plants of 8012-5, 8012-11 (VIT1 transgenic plants) and 8023-9, 8023-15 (IRT1 + FER1 transgenic plants) and one each of the wild type and the empty-vector controls. Petioles were mounted alongside the stems. Sections were mounted and scanned simultaneously. Fluorescence data were analyzed with the dynamic analysis method to obtain elemental maps with the software GeoPIXE v7.1. The incident flux was calibrated with reference films of known weight concentration, which were used to calculate elemental-weight concentrations from XRF peak areas via a fundamental-parameters approach.

Determination of total linamarin concentration in field-grown storage roots. Total linamarin concentrations were assessed in field-grown storage roots. Approximately $10 \mathrm{mg}$ of peeled storage-root tissue was lyophilized to a dry powder and extracted with buffer containing $100 \mathrm{mg} / \mathrm{mL} 2 \%$ acetonitrile, $1 \% \mathrm{HCOOH}$ and $10 \mu \mathrm{M}$ phenyl- $\beta$-D-glucoside by vortexing for $15 \mathrm{~min}$ at room temperature. Samples was centrifuged to remove debris and filtered through an $0.8-\mu \mathrm{m}$ polyethersulfone spin filter. One microliter of each sample was injected, and reversed-phase LCMS/MS was performed with a $0.5 \times 100-\mathrm{mm}$ polymeric reversed phase (PLRPS) column and $0.1 \% \mathrm{HCOOH}$ in water and acetonitrile as solvents. Sodiated linamarin and $\beta$-glucopyranoside (Sigma) were used as internal standards.

Mineral retention in foodstuffs generated from processed storage roots. Retention studies was performed on boiled storage roots and processed gari and fufu samples prepared from field-grown transgenic storage roots. Three biological replicates from five independent transgenic lines of each VIT1 and IRT1 + FER 1 storage roots were assessed. Approximately $10 \mathrm{~g}$ of fresh peeled storage root tissues/ line/replicate was chopped and boiled in $200 \mathrm{~mL}$ MQ water for 10-12 min. Boiled samples were patted dry with paper towels, lyophilized and digested for ICP-OES elemental analysis. Processing of storage roots for gari and fufu food products was performed as described earlier ${ }^{23}$. For gari preparation, storage root (150-200 $\mathrm{g}$ fresh weight) was peeled and grated, fermented in sacks and pressed with a hydraulic jack between wooden platforms to remove excess liquid from the pulp. Dewatered and fermented pulp was dried at room temperature for $48-72 \mathrm{~h}$, then forced through a 16-inch sieve mesh $(10 \mathrm{~mm})$ (Winco Industries). Fine pulp was roasted in a griddle at approximately $275^{\circ} \mathrm{C}$ for $10-15 \mathrm{~min}$ until the gari turned light brown. Fufu preparation was achieved by steeping $150-200 \mathrm{~g}$ fresh peeled roots in water for 3-4 d. Tissues were grated and blended in a food processor (Magic Bullet, Homeland Housewares), then pressed with a hydraulic jack between wooden platforms to remove water. Samples were dried in an oven at $90{ }^{\circ} \mathrm{C}$ for $24 \mathrm{~h}$ and milled to a powder with a food blender. Gari and fufu along with uncooked storage root samples were subjected to ICP-OES elemental analysis.

Bioaccessibility studies. Uncooked field-grown transgenic and wild-type cassava storage roots, plus gari, fufu and control samples were subjected to bioaccessibility studies. The bioaccessibility assays were performed in triplicate with a model simulating the digestive process in the mouth, stomach (gastric digestion) and small intestine (intestinal digestion). Enzyme solutions used for the in vitro digestion were prepared according to Glahn et al. ${ }^{39}$, with some modifications. Shortly before use, $\alpha$-amylase, pepsin and pancreatin/bile solutions were prepared separately as follows. (i) For $\alpha$-amylase solution, $\alpha$-amylase, Chelex-100 resin and $140 \mathrm{mM} \mathrm{NaCl}$ were mixed in a ratio of $1.0 \mathrm{~g} / 2.5 \mathrm{~g} / 15 \mathrm{~mL}$, respectively. (ii) to For pepsin solution, $208 \mathrm{mg}$ of pepsin and $1.9 \mathrm{~g}$ of Chelex-100 resin were dissolved in $7.5 \mathrm{~mL} 0.1 \mathrm{M} \mathrm{HCl}$. (iii) For pancreatin/bile solution, $30 \mathrm{mg}$ pancreatin, $150 \mathrm{mg}$ bile and $3.5 \mathrm{~g}$ Chelex-100 resin were dissolved in $7.5 \mathrm{~mL} 0.6 \mathrm{M} \mathrm{NaHCO}_{3}$. Each solution was mixed by being kept at room temperature for $30 \mathrm{~min}$ with vortexing every $5 \mathrm{~min}$. Solutions were filtered by gravity flow through a $30-\mu \mathrm{m}$ mesh filter to remove the Chelex-100 resin from the solution, and each filtrate was recovered. Chelex treatments were used to decrease the background levels of minerals associated with the enzyme reagents. The reagents $\alpha$-amylase $(1.5$ units/mg protein), porcine pepsin (800-2,500 units/mg protein), porcine bile extract and pancreatic enzymes ( $4 \times$ USP) were obtained from Sigma-Aldrich. Chelex-100 resin was obtained from Bio-Rad. In vitro digestion was performed according to Glahn et al. ${ }^{39}$ with some modifications. Simulation of digestion in the mouth was performed in a centrifuge tube by addition of $9 \mathrm{~mL}$ of $\alpha$-amylase solution to $1 \mathrm{~g}$ of freeze-dried sample; the mixture was incubated for $10 \mathrm{~min}$ at room temperature. Simulation of digestion in the stomach was carried out by addition of $10 \mathrm{~mL} 140 \mathrm{mM} \mathrm{NaCl}, 5 \mathrm{~mL}$ of $5 \mathrm{mM} \mathrm{KCl}$ and $1 \mathrm{~mL}$ of pepsin solution to the prior mixture, and the $\mathrm{pH}$ of the solution was adjusted to 2 by addition of concentrated $\mathrm{HCl}$. This mixture was kept in an incubator shaker at $37^{\circ} \mathrm{C}$ at 260 r.p.m. for $2 \mathrm{~h}$, and simulation of intestinal digestion was carried out in the same tube by addition of $1 \mathrm{~mL}$ of pancreatin/bile solution and $0.6 \mathrm{M} \mathrm{NaHCO}_{3}$ until the $\mathrm{pH}$ reached 5.7. The mixture was incubated for $2 \mathrm{~h}$ at $37^{\circ} \mathrm{C}$ and then placed into an ice bath to stop the digestion. The tube was centrifuged at 3,750 r.p.m. for $30 \mathrm{~min}$, and approximately $21 \mathrm{~mL}$ of supernatant was recovered. The recovered supernatant was split into two samples of $10 \mathrm{~mL}$, each aliquot was dried down to approximately $1 \mathrm{~mL}$, and samples were processed for elemental analysis. The semidried samples were predigested with $1 \mathrm{~mL}$ concentrated $\mathrm{HNO}_{3}$ for $16 \mathrm{~h}$ at room temperature in 150-mL digestion tubes, and most of the $\mathrm{CO}_{2}$ was allowed to bubble out of the solution. An additional $2 \mathrm{~mL}$ of concentrated $\mathrm{HNO}_{3}$ was added, and samples were heated for $1 \mathrm{~h}$ at $90^{\circ} \mathrm{C}$, then at $125^{\circ} \mathrm{C}$ until fuming stopped, after which $1.5 \mathrm{~mL}$ of $\mathrm{H}_{2} \mathrm{O}_{2}$ was added, and the sample was maintained at $125^{\circ} \mathrm{C}$ for $1 \mathrm{~h}$. This process was repeated an additional time, and samples were then dried at $200^{\circ} \mathrm{C}$, and the residues were dissolved in $7 \mathrm{~mL}$ of $2 \% \mathrm{HNO}_{3}$. Mineral concentrations were determined by ICP-OES (Ciros ICP- FCE12). Certified standards were used to calibrate the ICP-OES instrument. 'Blank' samples, consisting of digestion solutions with no cassava material, were used to determine background levels of minerals from the bioaccessibility solutions. Duplicate $0.5 \mathrm{~g}$ (dry weight) aliquots of each of the uncooked, gari or fufu samples were digested and analyzed by ICP-OES as described above, to determine the starting mineral concentrations of each sample. Duplicate mineral concentrations were averaged and converted to an average content ( $1 \mathrm{~g}$ basis). Mineral concentrations in the duplicate supernatant samples were averaged and multiplied by the number of milliliters of digestion solution (sum of all volumes added in the bioaccessibility assay) and converted to average mineral amounts released from each sample (normalized to a $1 \mathrm{~g}$ basis). Bioaccessibility percentages were then calculated with these content values: percentage bioaccessibility $=($ amount of each mineral released $\times 100) /$ starting content of each mineral. Triplicate aliquots of each food sample (uncooked root, gari or fufu) were used to calculate a mean and s.d. for each sample.

Determination of EARs. The EAR is a commonly used method to determine and express the average daily nutrient intake estimated to meet requirements of half the healthy individuals in a particular life stage and sex group within a given population. Variables that contribute to nutrient intake include the amount of food consumed per day, nutrients retained after boiling/cooking, proportion of nutrients absorbed and additional nutrient concentration present in the biofortified food. Nutrient requirements depend on the age and sex of the target population. The EAR was determined by dividing the physiological requirement of the nutrient by the fractional nutrient absorption. The potential nutritional contribution of iron-biofortified cassava lines toward the EARs for different demographic groups were calculated on the basis of assumptions made from food consumption ${ }^{28}(66.7 \mathrm{~g}$ $\mathrm{DW} / \mathrm{d}$ ), nutrient retention (70\%), absorbed proportion (5\%) and nutrient required $(460 \mu \mathrm{g})$ for children 1-3 years of age; on the basis of assumptions made from food consumption (116.7 g DW/d), nutrient retention (70\%), absorbed proportion (5\%) and nutrient required $(500 \mu \mathrm{g})$ for children 4-6 years of age; and on the basis of assumptions made from food consumption $(233.3 \mathrm{~g} \mathrm{DW} / \mathrm{d})$, nutrient retention (70\%), absorbed proportion (5\%) and nutrient required $(1,460 \mu \mathrm{g})$ for nonlactating, nonpregnant women (Fig. $4 \mathrm{a}, \mathrm{c}$ ). Zinc-biofortified cassava lines showing the potential nutritional contribution toward the EARs for different demographic groups were calculated on the basis of assumptions made from food consumption (66.7 $\mathrm{g} \mathrm{DW} / \mathrm{d})$, nutrient retention (70\%), absorbed proportion $(15 \%)$ and nutrient required $(740 \mu \mathrm{g})$ for children $1-3$ years of age; on the basis of assumptions made from food consumption (116.7 $\mathrm{g} \mathrm{DW} / \mathrm{d})$, nutrient retention (70\%), absorbed proportion $(15 \%)$ and nutrient required $(1200 \mu \mathrm{g})$ for children $4-6$ years of age; and on the basis of assumptions made from food consumption $(233.3 \mathrm{~g} \mathrm{DW} / \mathrm{d})$, nutrient retention $(70 \%)$, absorbed proportion $(15 \%)$ and nutrient required $(2,500 \mu \mathrm{g})$ for nonlactating, nonpregnant women ${ }^{40,41}$ (Fig. 4b,d). Nutrient absorption was calculated by multiplication of the food consumed per day with the additional nutrient concentration, nutrient retention and absorbed proportion of the food ${ }^{6}$.

Statistical analysis. The construction of graphics and the statistical analysis were performed with R software, version 3.4 .0 (ref. ${ }^{42}$ ). Box-and-whisker plots were constructed with the R package ggplot 2 (ref. ${ }^{43}$ ). The upper whisker extends from the hinge to the largest value no further than $1.5 \times$ the IQR from the hinge (where IQR is the distance between the first and third quartiles). The lower whisker extends from the hinge to the smallest value, at most $1.5 \times$ the IQR of the hinge. Data beyond the end of the whiskers were deemed outlying points and are plotted individually. To generate $P$ values as indicated in the graphics, a two-sample $t$ test was performed, comparing the wild type to the transgenic lines, unless otherwise stated.

Reporting Summary. Further information on research design is available in the Nature Research Reporting Summary linked to this article.

\section{Data availability}

The datasets generated and/or analyzed during the current study are available from the corresponding author on reasonable request. All data generated or analyzed during this study are included in this published article and its supplementary information files.

\section{References}

32. Beyene, G. et al. A virus-derived stacked RNAi construct confers robust resistance to cassava brown streak disease. Front. Plant Sci. 7 , 2052 (2017).

33. Cartwright, D. A., Brady, S. M., Orlando, D. A., Sturmfels, B. \& Benfey, P. N. Reconstructing spatiotemporal gene expression data from partial observations. Bioinformatics 25, 2581-2587 (2009). 
34. Chauhan, R. D., Beyene, G., Kalyaeva, M., Fauquet, C. M. \& Taylor, N. Improvements in Agrobacterium-mediated transformation of cassava (Manihot esculenta Crantz) for large-scale production of transgenic plants. Plant Cell Tiss. Org. 121, 591-603 (2015)

35. Taylor, N. et al. A high-throughput platform for the production and analysis of transgenic cassava (Manihot esculenta) plants. Trop. Plant Biol. 5, 127-139 (2012).

36. Farnham, M., Keinath, A. \& Grusak, M. Mineral concentration of broccoli florets in relation to year of cultivar release. Crop Sci. 51, 2721-2727 (2011).

37. Huang, R. et al. Application of CHESS single-bounce capillaries at synchrotron beamlines. J. Phys. Conf. Ser. 493, 012034 (2014).

38. Ryan, C. G. et al. Maia X-ray fluorescence imaging: capturing detail in complex natural samples. J. Phys. Conf. Ser. 499, 012002 (2014).
39. Glahn, R. P., Cheng, Z., Welch, R. M. \& Gregorio, G. B. Comparison of iron bioavailability from 15 rice genotypes: studies using an in vitro digestion/ caco-2 cell culture model. J. Agric. Food. Chem. 50, 3586-3591 (2002).

40. Institute of Medicine (US) Panel on Micronutrients. DRI: Dietary Reference Intakes for Vitamin A, Vitamin K, Arsenic, Boron, Chromium, Copper, Iodine, Iron, Manganesse, Molybdenum, Nickel, Silicon, Vanadium, and Zinc 42-501 (National Academy Press, Washington, DC, 2001).

41. HarvestPlus. Human Zinc Requirements: Report of the HarvestPlus Consultation on Physiological and Dietary Zinc Requirements (HarvestPlus, Rockville, MD, 2012).

42. R Core Team. R: a language and environment for statistical computing. R-Project 3, 201 (2013).

43. Wickham, H. ggplot2: Elegant Graphics for Data Analysis 2nd edn. (Springer, New York, 2016). 


\section{Reporting Summary}

Nature Research wishes to improve the reproducibility of the work that we publish. This form provides structure for consistency and transparency in reporting. For further information on Nature Research policies, see Authors \& Referees and the Editorial Policy Checklist.

\section{Statistical parameters}

When statistical analyses are reported, confirm that the following items are present in the relevant location (e.g. figure legend, table legend, main text, or Methods section).

n/a Confirmed

$\bigotimes$ The exact sample size $(n)$ for each experimental group/condition, given as a discrete number and unit of measurement

$\searrow$ An indication of whether measurements were taken from distinct samples or whether the same sample was measured repeatedly

$\triangle$ The statistical test(s) used AND whether they are one- or two-sided

Only common tests should be described solely by name; describe more complex techniques in the Methods section.

Х $\square$ A description of all covariates tested

Х $\square$ A description of any assumptions or corrections, such as tests of normality and adjustment for multiple comparisons A full description of the statistics including central tendency (e.g. means) or other basic estimates (e.g. regression coefficient) AND variation (e.g. standard deviation) or associated estimates of uncertainty (e.g. confidence intervals)

For null hypothesis testing, the test statistic (e.g. $F, t, r$ ) with confidence intervals, effect sizes, degrees of freedom and $P$ value noted

Give $P$ values as exact values whenever suitable.

Х $\square$ For Bayesian analysis, information on the choice of priors and Markov chain Monte Carlo settings

Х $\square$ For hierarchical and complex designs, identification of the appropriate level for tests and full reporting of outcomes

Х $\square$ Estimates of effect sizes (e.g. Cohen's $d$, Pearson's $r$ ), indicating how they were calculated

Clearly defined error bars

State explicitly what error bars represent (e.g. SD, SE, CI)

Our web collection on statistics for biologists may be useful.

\section{Software and code}

Policy information about availability of computer code

Data collection

R software, version 3.4.0 and GeoPIXE v7.1 for X-ray fluorescence (XRF)

Data analysis

Construction of graphics and statistical analysis were performed using R software, version 3.4.0. Box and whisker plots were constructed using the R package "ggplot2". To generate $p$-values as indicated in the graphics, a two-sample t-test was performed comparing the wildtype to the transgenic lines unless otherwise stated. X-ray fluorescence (XRF) data was analyzed by the dynamic analysis (DA) method to obtain elemental maps using the software GeoPIXE v7.1. The incident flux was calibrated using reference films of known weight concentration, used to calculate elemental weight concentrations from XRF peak areas via a fundamental-parameters approach. 
Policy information about availability of data

All manuscripts must include a data availability statement. This statement should provide the following information, where applicable:

- Accession codes, unique identifiers, or web links for publicly available datasets

- A list of figures that have associated raw data

- A description of any restrictions on data availability

The datasets generated and/or analyzed during the current study are available from the corresponding author on reasonable request. All data generated or analysed during this study are included in this published article and its supplementary information files.

\section{Field-specific reporting}

Please select the best fit for your research. If you are not sure, read the appropriate sections before making your selection.

\ Life sciences $\quad \square$ Behavioural \& social sciences $\quad \square$ Ecological, evolutionary \& environmental sciences

For a reference copy of the document with all sections, see nature.com/authors/policies/ReportingSummary-flat.pdf

\section{Life sciences study design}

All studies must disclose on these points even when the disclosure is negative.

Sample size For all the greenhouse and field experiments, sample size were determined with statistical rigor

Data exclusions For VIT1 transgenic plants, one transgenic event was excluded due to poor growth performance in the field and had very small amount of roots.

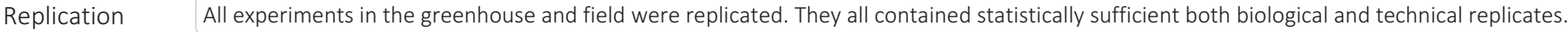

Randomization Whenever possible in the field experiments, plants were tested in a randomized replicated trial. In other experiments, plants were allocated in a random way to minimize edge effects.

Blinding $\quad$ All the mineral measurements (ICP) were done by a in-house/third party lab and they were not aware of the sample name and number. During processing and collecting data in the greenhouse, technicians also were not aware of the sample name and number.

\section{Reporting for specific materials, systems and methods}

Materials \& experimental systems

$\mathrm{n} / \mathrm{a}$ Involved in the study

$\searrow \square$ Unique biological materials

Х $\square$ Antibodies

Х Eukaryotic cell lines

Х $\square$ Palaeontology

Х $\square$ Animals and other organisms

$\backslash \square$ Human research participants

\begin{tabular}{l|l}
\multicolumn{2}{l}{ Methods } \\
\hline n/a & Involved in the study \\
$\square$ & $\square$ ChIP-seq \\
$\triangle$ & $\square$ Flow cytometry \\
$\triangle$ & $\square$ MRI-based neuroimaging
\end{tabular}

\section{Eukaryotic cell lines}

Policy information about cell lines

Cell line source(s)

Authentication

Mycoplasma contamination 